\title{
Regiões, cidades e comunidades resilientes: novos princípios de desenvolvimento
}

\author{
Resilient regions, cities and communities: new development principles
}

Carlos Gonçalves

Universidade de Lisboa, Instituto de Geografia e Ordenamento do Território, Centro de Estudos Geográficos, Lisboa, Portugal

\section{Resumo}

Os efeitos da sucessão/sobreposição de crises ambientais, de insegurança, de governação, financeiras, econômicas e sociais diluíram-se no quotidiano de boa parte das regiões do planeta. Essa presença constante destrói a estabilidade e a previsibilidade associadas aos estádios mais avançados de progresso. A turbulência deixou de ser exceção e passou a ser regra. Neste artigo, discute-se o que vulnerabiliza os territórios e capacita as regiões, as cidades e as comunidades para recuperá-las, quando expostas a crises agudas e/ou prolongadas. Procuram-se justificações para o fato de alguns territórios falharem no esforço de retomar a trajetória pré-crise, enquanto outros cumprem ou superam esse objetivo, robustecendo-se no decurso dos episódios desestabilizadores. Neste artigo, observamos o desenvolvimento regional, urbano e local por meio do paradigma da resiliência evolutiva, identificando os gatilhos e as condições para a sustentabilidade do desenvolvimento, muito para além da lógica reativa de manter, distender ou tentar retomar hipotéticos equilíbrios vigentes pré-crise.

Palavras-chave: Região resiliente. Cidade resiliente. Comunidade resiliente.

\section{Abstract}

Succession/overlap effects of environmental, insecurity, governance, financial, economic and social crises have been diluted in the daily life of great part of the planet regions. The turbulence is no longer the exception and has become the rule. It is important, therefore, to discuss what weakens the territories and what empowers the regions, cities and communities to recover when they are exposed to acute and/or prolonged crises. It urges to seek justifications to the fact that some territories fail the effort to reclaim the pre-crisis trajectory, while others, meet or exceed this goal, getting stronger in the destabilizing episodes course. In this article we observe the regional, urban and local development through the paradigm of evolutionary resiliency. This allows to identify the triggers and the conditions for the development sustainability, far beyond the reactive logic to keep, overstrain or try to reclaim hypothetical effective balances pre-crisis.

Keywords: Resilient region. Resilient city. Resilient community.

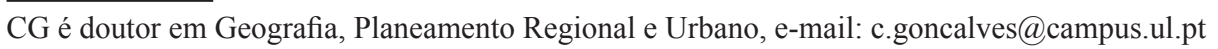




\section{Introdução}

A pressão sobre os limites de funcionamento dos sistemas ambientais, o aumento das desigualdades e a estenose na acessibilidade a recursos básicos bloqueiam as trajetórias de desenvolvimento, desligando-as da sustentabilidade. Com as crises recentes, as regiões, as cidades e as comunidades estão mais expostas a vulnerabilidades resultantes das dívidas soberanas, da instabilidade do sistema financeiro, dos desequilíbrios dos modelos de crescimento, da falta de emprego e da inconsistência das estruturas de governança (ONU, 2012).

O sentido das conclusões de autores, os quais perspectivam a resiliência das cidades pelo comportamento que estas manifestam quando expostas a fenômenos extremos (Berke \& Campanella, 2006; Vale \& Campanella, 2005; Pendall et al., 2010), aponta para a necessidade de se retomar os modelos pré-crise.

Considerando como ponto de partida a definição de Holling (2001) e Alberti et al. (2003), a resiliência urbana traduz a medida das alterações desencadeadas e toleradas pelas cidades, antes de se reorganizarem integralmente em torno de novas estruturas e processos. As reconfigurações que a definição de resiliência foi sofrendo decorrem do esforço de aplicação prática de disciplinas externas à física dos materiais ou à engenharia, construindo outros significados desvinculados do comportamento de estruturas construídas: resistência pela flexibilidade.

As definições de resiliência permitem abordagens descritivas, atendendo: à "[...] medida da persistência e capacidade dos sistemas para absorver mudanças e perturbações mantendo a população e a estrutura de relações (ou entre variáveis de estado) [...]" (Holling, 1973, p. 14); à "[...] magnitude de perturbação que podem ser absorvidas, alterando as variáveis e os processos que controlam o seu comportamento, antes que o sistema mude a sua estrutura [...]" (Holling \& Meffet, 1996, p. 4); ou à "[...] capacidade de um sistema experienciar choques mantendo, essencialmente, a mesma função, estrutura, reações, e portanto, preservando a identidade [...]" (Walker \& Salt, 2006, p. 2).

Outro conjunto de definições aponta para as capacidades que os territórios devem demonstrar: “[...] para absorver perturbações, para gerar auto-organização e para desencadear aprendizagem e adaptação [...]" (Walker et al., 2002); para clarificar domínios de atuação, "[...] latitude (largura do domínio), resistência (altura do domínio), precariedade, relações entre escalas [...]" (Folke et al., 2004, p. 573); para propor dimensões operacionais, "resiliência do quê e para quê?" (Carpenter et al., 2001); para valorizar a "[...] capacidade que o sistema demonstra de manter a sua identidade em quadros de perturbações, mudança e choques internos e externos [...]" (Cumming et al., 2005, p. 976).

No entanto, surgem autores que privilegiam aspectos sociais, associados à "[...] capacidade que grupos ou comunidades manifestam para lidar com tensões e distúrbios externos, resultantes de mudanças sociais, políticas e ambientais [...]" (Adger, 2000, p. 347). Ou então, de um modo mais abrangente, consideram a relação entre sociedade e economia, calculando a "[...] probabilidade de transição entre estados, como função do balanço entre atividades de produção e padrões de consumo, condicionada pela ação dos decisores [...]" (Brock et al., 2002, p. 273), e a "[...] capacidade presente no sistema para suportar choques no funcionamento do mercado ou na estrutura ambiental, sem comprometer a eficiência na distribuição de recursos [...]" (Perrings, 2006, p. 418).

Propõem-se também abordagens híbridas, tais como as que conjugam a "[...] capacidade intrínseca que o ecossistema apresenta para manter os serviços ambientais desejados, mesmo em conjunturas ambientais instáveis induzidas pelas atividades humanas [...]" (Folke et al., 2002, p. 14), a "[...] capacidade que um sistema socioecológico apresenta para absorver perturbações recorrentes [...] mantendo as estruturas essenciais, os processos e os feedbacks [...]" (Adger et al., 2005 , p. 1036), e as "[...] propriedades quantitativas que, nos ecossistemas, mudam de modo dinâmico em todos os níveis da hierarquia [...]" (Holling, 2001).

Em outro plano, a resiliência territorial é vista como "[...] perspetiva ou abordagem, capaz de analisar sistemas socioecológicos [...]" (Folke, 2006, p. 260), como "[...] flexibilidade projetada no longo prazo [...]" (Pickett et al., 2004, p. 381) ou como "[...] capacidade de manutenção, a longo prazo, de um dado capital natural [...]" (Ott \& Döring, 2004, p. 213).

De modo simplificado, resiliência regional, urbana ou local varia entre a competência que um sistema socioeconômico demonstra para se recuperar de um choque ou de uma disrupção, a possibilidade que uma economia manifesta para manter o mesmo rumo durante uma crise e a capacidade que apresenta, 
quando exposta a um choque, para recuperar o nível de desenvolvimento prévio e/ou de reposicioná-lo em um patamar superior. Esse reposicionamento pode ser observado no padrão de acesso ao rendimento, no saldo demográfico ou nos níveis de coesão e de qualidade de vida (Dawley et al., 2010).

As noções de adaptação, adaptabilidade e capacidade adaptativa ajudam a clarificar o entendimento da resiliência regional, urbana ou local. Adaptação é entendida como resposta a uma crise para retornar a um modelo de desenvolvimento que vigorava no período pré-crise. Adaptabilidade materializa o alinhamento com oportunidades, opções e decisões conducentes a abandonar uma estrutura de funções, não obstante ela ter sido geradora de sucesso. Orienta o território para outra trajetória, explorando novos nichos de progresso. Essa vertente da resiliência manifesta-se na tolerância e na abertura para lidar com incertezas, com ineficiências e com os riscos de impopularidade associados à procura de modelos alternativos. Esses posicionamentos correspondem a diferentes fórmulas de resiliência territorial. A relação entre essas competências compõe um emaranhado de relações entre setores, destes com as geometrias do mercado de trabalho, com as configurações de capital ambiental, social, cívico e com os arranjos de poder. Da conjugação de fatores resultam fórmulas únicas de resiliência observáveis em territórios concretos (Dawley et al., 2010).

São exemplos de diálogos entre adaptação e adaptabilidade: a transição operada na região de Massachusetts de uma estrutura em declínio baseada nos têxteis para uma matriz econômica assente em tecnologia de ponta, ao longo da "Route 128"; a reconfiguração verificada na região do Ruhr, deslocando-se para modalidades "limpas" de exploração de carvão e para indústrias relacionadas com o ambiente; a reformulação da estrutura industrial de Toledo, que era obsoleta, atraindo novas oportunidades, por exemplo, nas áreas das energias renováveis; ou ainda o redesenho de uma estrutura econômica, urbana e social historicamente embrenhada em indústrias pesadas, em Bilbau (Dawley et al., 2010).

Importa, pois, saber como é que a perspectiva evolucionista capta as variações regionais, urbanas e locais de capacidade de adaptação e de adaptabilidade. A resiliência regional, próxima da capacidade adaptativa, sinaliza as diferenças manifestas pelas regiões para gerir mudanças constantes ou choques repentinos na sua competitividade, na organização dos mercados, no nível de internalização de inovações tecnológicas, na aptidão das estruturas políticas e respectivas condições que dinamizam, ou que bloqueiam, a trajetória evolutiva de um sistema socioeconômico ao longo dos tempos (Simmie \& Martin, 2010).

Tendo por base a revisão da literatura, nas três seções do artigo, procuramos definir, com mais detalhe, quais os princípios que norteiam a resiliência regional, urbana e local. Com a conjugação das três aproximações à resiliência territorial, pretendemos contribuir para a discussão dedicada à definição de novos princípios que reforcem a meta da sustentabilidade vista enquanto desígnio civilizacional.

\section{Regiões resilientes}

Os estudos de resiliência regional captam o modo como as comunidades reagem e recuperam-se de disrupções que interferem com o seu processo de desenvolvimento. Essa missão, próxima da interpretação feita em ecologia, é complementada pela possibilidade de se identificarem saltos nos percursos de desenvolvimento, deslocando uma trajetória pré-crise para outro modelo, ritmo ou arranjo de funções: resiliência evolutiva.

De modo simplificado, identificam-se três tipologias de resiliência regional: pela resistência, pela reposição e pela superação. No primeiro caso, a região consegue contornar crises porque a sua estrutura socioeconômica é de tal modo robusta, que os efeitos são negligenciáveis ou não são sentidos. A análise incide na magnitude das crises e na capacidade de absorção dos seus efeitos pelas estruturas socioeconômicas. No segundo caso, a região regride, mas tem elasticidade suficiente para repor o ritmo de crescimento anterior, colocando-se apenas em coação o tempo despendido para que tal ocorra. Na última das três tipologias, a região consegue, para além de absorver os efeitos de maior magnitude e de repor o padrão de prosperidade, reposicionar o modelo de desenvolvimento precedente, fortalecendo-o. Nesse caso, assume-se que o paradigma anterior não produzia os efeitos desejados ${ }^{1}$, e a região apresenta competências para superar não só um quadro de

\footnotetext{
${ }^{1}$ Nomeadamente: a geração e distribuição de riqueza, a acessibilidade racional a recursos, a construção de capital social e ecológico, a criação e sustentação de qualidade de vida.
} 
recessão, mas também um contexto de desenvolvimento inadequado.

Esses desempenhos têm lugar em diferentes escalas ou delimitações setoriais. A existência de condições para desencadear cada um dos comportamentos referidos manifesta-se no mercado de trabalho e nos aspectos infraestruturais, ou ainda por referência a rupturas sociais concretas (um surto epidêmico ou um acidente que dizima parte da população). É nesse plano que se materializa a natureza dinâmica e complexa dos territórios resilientes, organizados para gerar persistência, adaptabilidade e ganhos no capital de transmutabilidade.

Sujeitas a crises, as regiões mais resilientes são encorajadas a desencadear transformações, compondo novas estruturas de desenvolvimento, tirando partido de caminhos alternativos apontados para nichos que sinalizam possibilidades inexploradas (ESPON, 2012). É nesse plano evolutivo que Martin (2012) enxerta a ideia de histerese, associando-a a mudanças contínuas na estrutura econômica de uma região.

Nos casos em que os choques se apresentam com grande severidade, pode alterar-se o quadro em que se movimentam os agentes económicos e até mesmo a arquitetura setorial, reencaminhando a economia para uma nova rota de progresso [...] (Martin, 2012, p. 8).

Daqui resulta uma divisão entre "histerese positiva" e "histerese negativa", de acordo com o reposicionamento do patamar de desenvolvimento pós-crise. Este, pode fixarse em um nível inferior ao que existia (por exemplo, no que tange às oportunidades de acesso a rendimentos) ou catapultar o sistema socioeconômico para uma configuração sustentadora de níveis de progresso superiores aos que se verificavam antes do período desestabilizador. A interpretação de Martin (2012) permite que se tipifique a qualidade da resposta que um território desencadeia perante uma crise. Serve, ainda, para estremar os planos de resiliência indesejada de outras que possuem pendor mais profícuo. Em outras palavras, identificam-se fatores que contribuem para perpetuar situações de insustentabilidade e modelos de crescimento que produzem iniquidade, disfuncionalidades e convulsões sociais. Resiliência regional congrega elementos que viabilizam resistência com outros que facilitam recuperação, reorientação e renovação. É esta última dimensão que abre a possibilidade de um território reassumir um novo trajeto de desenvolvimento distinto do que conduziu à(s) crise(s).
A necessidade de fixar a concepção de resiliência de um território específico obriga a determinação de suas fronteiras e que se situe o intervalo temporal a considerar (Pendall et al., 2010). As regiões, especialmente se as entendermos a partir dos sistemas urbanos que as suportam, integrando as concepções de região urbana policêntrica (Robert, 2001) e de cidade-região (Parr, 2014), operam em múltiplas escalas, porquanto o seu arranjo de funções se encontra distribuído por um conjunto variado de localizações.

Entendidas como sistemas adaptativos complexos, as regiões urbanas policêntricas ou as cidades-região caracterizam-se por serem sistemas abertos, podendo manifestar níveis de conexão internos muito distintos. São sistemas que geram e recebem enormes fluxos de energia, matéria e informação, obrigando as suas estruturas internas (sociais, econômicas, biofísicas) a lidar com flutuações que tanto podem ser de progressão lenta como de manifestação repentina. Do processo de trocas constantes, amplificado pela sua natureza "não linear", resultam efeitos de feedback que, não raras vezes, deslocam o processo de desenvolvimento para estádios de irreversibilidade. Esse tipo de propriedades leva em consideração que "[...] as regiões, tal como outros sistemas adaptativos complexos, são mais do que a soma das suas partes, por tal admitem, com dificuldade, os exercícios simplistas de modelação [...]" (Pendall et al., 2010, p. 78).

Relativamente à escala temporal, é necessário considerar que, mesmo nos casos em que a ponte entre resiliência, adaptação e sustentabilidade é fácil de estabelecer, quando se consideram percursos evolutivos de longo prazo, os trabalhos desenvolvidos, por exemplo, no campo da arqueologia, demonstram que respostas de perfil estratégico, aparentemente incrementadoras da resiliência, podem ser responsáveis por vulnerabilidade no longo prazo. Em casos extremos, encaminham componentes do sistema socioambiental para o colapso (Chelleri, 2012).

Em uma estrutura produtiva regional, a composição da matriz setorial assume centralidade na abordagem da resiliência e ajuda a explicar as diferenças de comportamento, quer se trate da capacidade para resistir a mudanças profundas, quer se orientem para reposição ou superação. Considerando a resistência como modelo, e tomando por base um determinado quadro de crise, importa saber que regiões foram primeiramente afetadas e que setores apresentaram maior profundidade e extensão dos efeitos, por 
exemplo, verificados na destruição de emprego. Essa abordagem à resiliência regional pode direcionar-se para aferir não o impacto, mas a capacidade de sair do quadro de crise ("bounce-back") e/ou de regressar ("comeback") à trajetória de desenvolvimento prévia. Essas duas dimensões incluídas nessa ligação com o desenvolvimento aproximam-se dos indicadores de estado ou de desempenho, na medida em que regiões com maior capacidade de resistência estarão mais habilitadas e com maior facilidade em retomar o sentido de desenvolvimento pré-crise.

Esses dois modelos, a partir dos quais se perspectiva a resiliência territorial, apesar de válidos do ponto de vista instrumental, orientam-se pelo racional de que se pode suster ou retomar uma posição de equilíbrio em uma trajetória de desenvolvimento, muito associada à elasticidade própria das estruturas criadas pela engenharia. Do ponto de vista instrumental, são abordagens válidas e úteis, contudo colocam de lado a possibilidade de equacionar a natureza do modelo que gerou e que sofreu a situação de crise. Desse modo, a análise da resiliência regional aproxima-se da perspectiva evolucionista, vista enquanto processo dinâmico com incrementos constantes, desligados dos mecanismos de estabilidade (Dawley et al., 2010). O referencial da resiliência evolutiva "[...] oferece ferramentas teóricas e empíricas novas, que extravasam as abordagens existentes [...]" (Dawley etal., 2010 , p. 3). Os avanços conseguidos evidenciam que as regiões mais dinâmicas são também as que apresentam maior capacidade de adaptação. Todavia ainda não é possível perceber as regiões afastadas dos centros mais dinâmicos, quando confrontadas com uma multiplicidade de mudanças estruturais, podem estimular adaptabilidade e desenvolver resiliência (Dawley et al., 2010).

Observando o desenvolvimento regional pela lente da resiliência, cria-se a possibilidade de reconfigurar as estruturas socioeconômicas que organizam um território para responder diante das situações de crise, ou seja, abre margem para planejar e organizar modelos de desenvolvimento valorizadores de estados de preparação e que diluam mecanismos de recuperação úteis na fase pós-choque. A Figura 1 esquematiza a situação de partida de uma região antes de um evento desestabilizador, a tipologia do próprio evento e os resultados que a partir dele se podem obter. Estabelecem-se relações entre a fonte da crise, a capacidade apresentada para suportar ou lidar com ela, a magnitude do impacto, a orientação da trajetória subsequente e os resultados possíveis (Dabson et al., 2012).

A capacidade de resiliência que a região manifesta é colocada em confronto com a vulnerabilidade inerente e com os recursos disponíveis para desencadear adaptabilidade. No impacto, convergem a severidade do choque e a capacidade de absorção, visíveis no nível de disfuncionalidade provocado. A trajetória

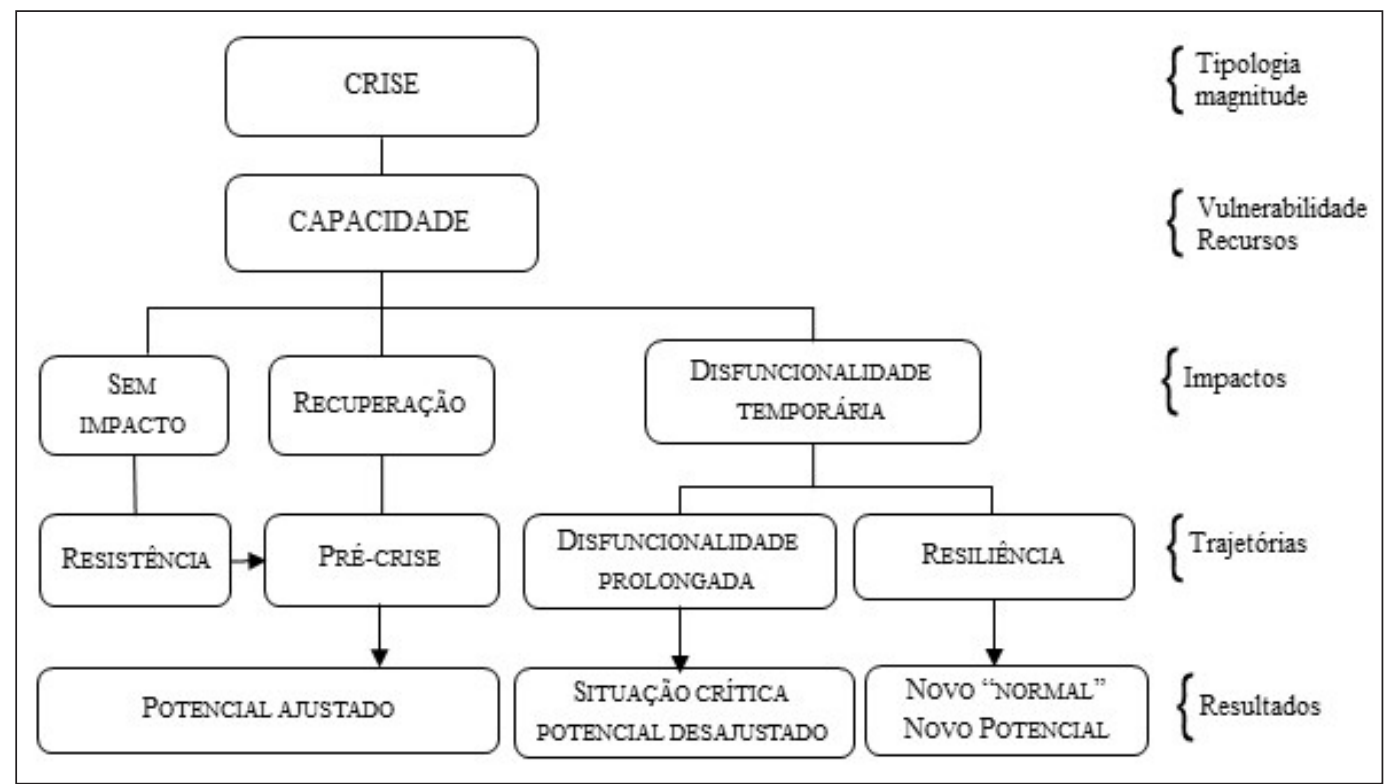

Figura 1 - Estrutura do racional da resiliência regional Fonte: Elaborado pelo autor. 
concretiza-se em quatro posições diferentes que podem ser assumidas pós-crise: resistência, recuperação, resiliência e disfuncionalidade prolongada. Os resultados podem ser de três tipos diferentes: a região permanece presa em uma situação crítica por um longo período de tempo; organiza-se em torno de um novo modelo (nova normalidade); ou retoma a configuração pré-crise.

A Figura 2 esquematiza cenários regionais e locais possíveis diante de um episódio de crise (A). Se a região apresenta recursos que lhe permitem lidar com uma situação de crise, de maneira que não se verifiquem perdas nas suas funções, quer dizer que o seu ponto de partida lhe permitiu resistir a esse tipo de crise (trajetória $\mathrm{C} 0$ ). Se a mesma crise provoca uma ruptura na sua capacidade de resistir, inicia-se uma fase em que se degradam as suas funcionalidades (retração do potencial socioeconômico do tipo B1). A natureza da fase seguinte depende da consistência dos recursos que apresenta. Nessa situação, a trajetória mais fácil é a que habilita a recuperação, ou seja, o retorno ao nível de funcionamento anterior sem que seja necessário mobilizar grandes movimentos de adaptação (trajetória C1). Esse processo pode ser designado de resiliência estática ou reposição.
Por uma trajetória diferente, outra região desencadeia um novo arranjo de funções, desenhando uma "nova normalidade" que corresponde a um incremento na matriz de funcionamento e nas oportunidades de desenvolvimento por referência ao período anterior ao surgimento da crise (trajetória C2 criando novo potencial socioeconômico B3). 0 pior cenário desenha-se quando a disfuncionalidade temporária se transforma em crônica (trajetória C3 implicando uma perda do potencial socioeconômico do tipo B2), mantendo a região em uma situação crítica e inviabilizando as suas possibilidades de retomar um nível de funcionamento mínimo aceitável (Dabson etal., 2012). Por esse caminho, retoma-se, inicia-se ou configura-se o que Naudé et al. (2009), Dempster (2007), Martin-Breen \& Anderies (2011), entre outros, sinalizam como armadilha da pobreza ("poverty trap"). Se o ciclo adaptativo colapsa devido à erosão do potencial de desenvolvimento e de reforço da diversidade, decorrentes de gestão inadequada ou por efeito de uma força externa, um estado de empobrecimento pode ser o resultado, caracterizado por baixa conectividade, baixo potencial e baixa resiliência, consumando-se em uma armadilha de pobreza (Holling, 2002, p. 95-96 apud Dempster, 2007, p. 23).

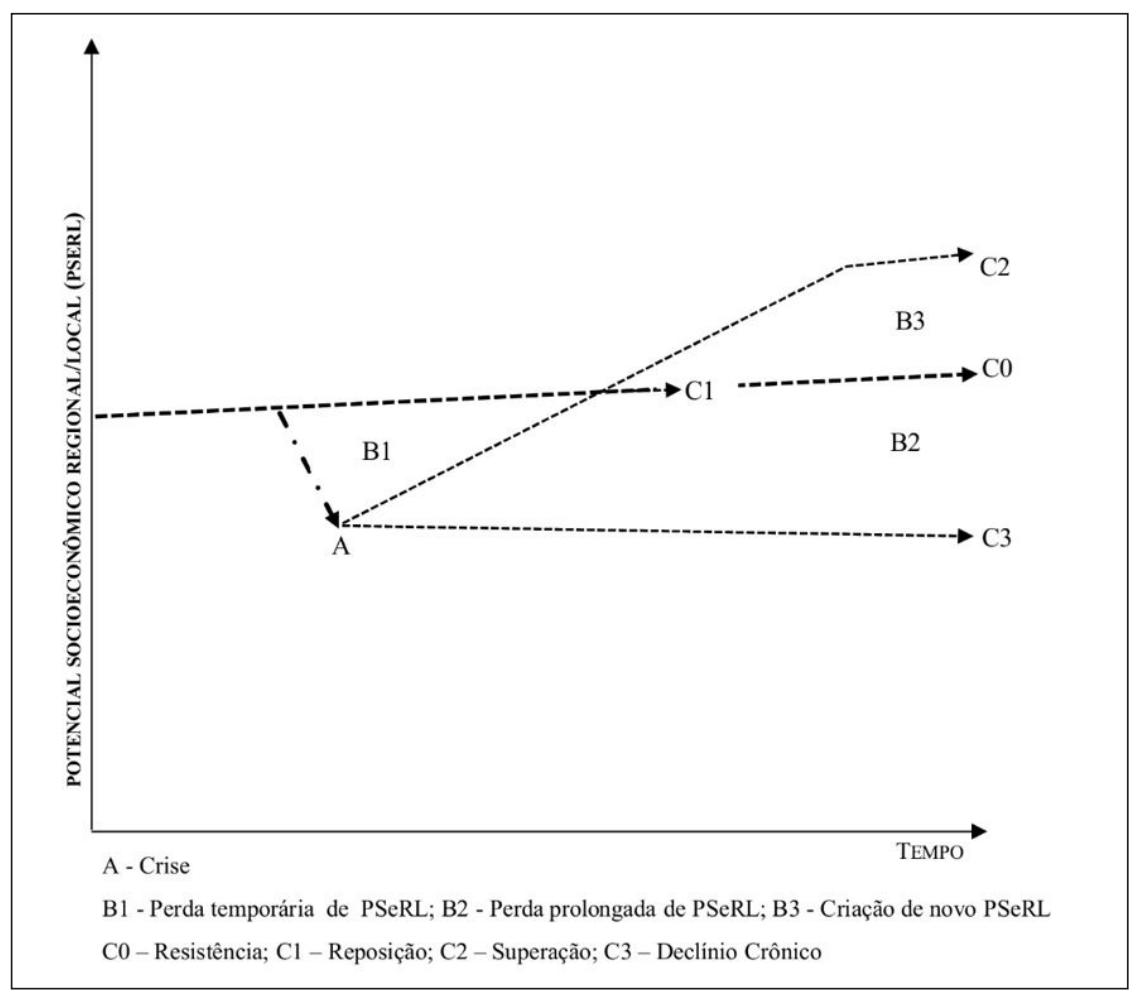

Figura 2 - Tipologias de trajetórias de contração/expansão da resiliênncia regional ou local Fonte: Elaborado pelo autor. 
Considerando a reflexão subjacente à Figura 2, uma região com elevada resiliência antecipa ou consegue mitigar ameaças e desenvolve uma visão de futuro que coloca a resiliência como prioridade, criando uma estrutura de planejamento que deixa em aberto caminhos de recuperação, permitindo-lhe, em um curto período de tempo, resgatar as funções principais e desencadear um percurso condizente a uma "nova normalidade". Confrontada com uma situação de crise, sofrerá algumas perdas (B1), mas a profundidade desse desgaste de funcionalidades será menos profunda do que a verificada na segunda situação representada (B2). Algumas regiões estarão habilitadas para retomar aos níveis de progresso pré-crise (trajetória C1); outras se organizam em torno de um modelo que não consegue atingir os padrões de qualidade de vida anteriores (trajetória C3); outras ainda, capacitadas para identificar novas oportunidades e para desencadear transformações, deslocam-se para um estádio de desenvolvimento superior ao que detinham antes do fenômeno desestabilizador (C2) (Dabson et al., 2012).

Não é expectável que uma região, uma cidade ou uma comunidade industrial, turística, pesqueira ou de qualquer outra natureza mantenha inalterada a capacidade de gerar e manter emprego sem que tenham mudanças estruturais mais ou menos contínuas. Regiões dotadas de mão de obra qualificada estão mais preparadas para desencadear as inovações que lhes permitem absorver, superar ou transformar-se no decurso de situações de crise (Ficenec, 2010). A consistência da cultura organizacional é outro aspecto relevante para a resiliência das regiões.

Vários autores prefiguram a resiliência regional pelos resultados que apresenta, por exemplo, na capacidade de criação de emprego, de aumento dos rendimentos do trabalho e de redução das iniquidades sociais (vistas, por exemplo, na ampliação ou na contração da classe média). Em outras palavras: outputs positivos no acesso a rendimentos e na coesão social associam-se à consolidação de desenvolvimento regional, transpondo-se para diferentes medidas de sucesso por referência ao tipo de desafio diante do qual a região se encontra colocada (Chapple \& Lester, 2010). Assim, a resiliência regional traduz-se nas "[...] competências de transformação dos outputs que uma região apresenta, face a um determinado desafio [...]" (Chapple \& Lester, 2010, p. 86).
Uma cultura pró-resilência implica sempre que se considere o peso das trajetórias do passado, ou seja, obriga que assimilem e reproduzam interpretações de "normalidades" e de crises vistas a longo prazo (Raco \& Street, 2012). Na escala mais próxima da componente humana dos territórios, essa competência coletiva pode traduzir-se em um conjunto de atributos associáveis a uma comunidade, a uma cidade ou a um sistema urbano. Atentamos, em seguida, à segunda das duas escalas enunciadas.

\section{Cidades e sistemas urbanos resilientes}

Na definição de cidade resiliente, mesclam-se as componentes física e humana. A parte estrutural, quando sujeita a situações disruptivas, tem de estar preparada para sobreviver e funcionar sob estresse. Desse modo, a cidade resiliente corresponde a uma rede sustentável de sistemas físicos e de comunidades humanas. Os sistemas físicos conjugam elementos construídos com outros de aspecto biofísico. Incluem-se aqui os corredores das estradas e ruas, os edifícios, as infraestruturas, as comunicações, as redes de distribuição de energia, mas também a rede de drenagem, os solos, a topografia, a geologia e outros sistemas naturais. Em outras palavras, “[...] os sistemas físicos funcionam como corpo da cidade, os seus ossos, artérias, músculos [...]" (Godschalk, 2003, p. 137). Associando as dimensões física e humana da cidade, $\mathrm{o}$ autor refere-se às comunidades humanas como sendo "[...] as componentes sociais e institucionais da cidade [...]". Incluem estruturas formais e informais, associações estáveis e outras ad hoc que impactam as áreas urbanas: escolas, bairros, agências, organizações, empresas, grupos de interesses etc. Em suma, "[...] as comunidades funcionam como o cérebro da cidade, direcionando as suas atividades, respondendo às suas necessidades e aprendendo com as suas experiências [...]" (Godschalk, 2003, p. 137). Resulta daqui a necessidade de integração entre o suporte físico da cidade e a sua componente humana, decantando o que confere resiliência à sua trajetória evolutiva.

Várias incursões aproximam a identificação de vulnerabilidades com os princípios inerentes à resiliência evolutiva. Cidade resiliente pode ser vista como sendo a que é capaz de gerar, nos seus sistemas, competências para lidar com ameaças à 
sua sobrevivência e recursos para se autossustentar. Ballard-Rosa (2010, p. 175), refletindo em torno do conceito, invoca o que chama de "abordagem histórica de banda larga de Mumford", conferindo-lhe validade quando diz que a cidade é um objeto de arquitetura, social, natural e cultural. Contudo, compreende que o trabalho de Henri Lefebvre complementa a definição de Mumford, de acordo com o qual a cidade é simultaneamente um espaço de representações e um espaço de representação. Ballard-Rosa (2010, p. 176) apresenta a sua definição de cidade resiliente valorizando a

[...] interface entre o ambiente natural e construído (materialidades), entre o fluxo de pessoas e mercadorias (mobilidades), mas também, entre representações espaciais conflituantes (memórias) e as centralizações de poder (divisão/segregação).

Essa definição é particularmente relevante, porque isola quatro dimensões do funcionamento da cidade: estruturas, fluxos, representações e relações de poder. Do confronto dessas forças ganha ênfase a evidência de que a cidade é um palco de contrastes e de constantes mudanças.

Podemos associar a definição de cidade resiliente, nas quatro dimensões ${ }^{2}$ enunciadas, à noção de metabolismo urbano. Nesse plano, Folke (1997) apud Resilience Alliance (2007, p. 11), nos diz que as populações urbanas dependem da produtividade dos ecossistemas nos quais as suas cidades estão integradas. Esses ecossistemas, para além das transferências de energia, disponibilizam "[...] bens materiais e serviços não-materiais, ambos alicerces do bem-estar e da qualidade de vida urbana [...]". A noção de sistema socioambiental convoca uma concepção de resiliência, por meio da qual se reforça a necessidade de integração sistêmica entre a componente social e a estrutura ecológica, posicionando-as no mesmo patamar.

O estudo da resiliência urbana encara as cidades como o exemplo de um sistema adaptativo complexo (Batty etal., 2004 apud Resilience Alliance, 2007; Martin, 2012; Pendall et al., 2010). Bai (2003), apud Resilience Alliance (2007), afirma que as cidades são sistemas vivos, dinâmicos, geradores de (egerados por) conectividades e abertos a constantes interações, quer no seu interior, quer por via da exposição a fatores externos.

\footnotetext{
$\overline{2}$ i) materialidades; ii) memórias; iii) palco de divisões/segregação; iv) estruturas, fluxos, representações e relações de poder.
}

A resiliência urbana não se circunscreve na função de resposta a perturbações, corporizada, por exemplo, na capacidade de recuperar do impacto de fenômenos extremos. Quando se aplica o referencial da resiliência aos sistemas socioambientais urbanos, considerando que os contextos de incerteza são constantes, percebe-se que quanto mais flexíveis e capazes de proceder a ajustamentos, mais habilitados estão a capitalizar as oportunidades. Em suma, as "competências" no sentido da resiliência, das quais se notam a flexibilidade para conviver com o inesperado e a adaptação, são elementos-chave para o futuro das cidades. A ideia de que as cidades estão sujeitas a pressões que, acumuladas, podem provocar rupturas é um ponto central na abordagem da geografia ao estudo da resiliência evolutiva.

A Resilience Alliance Initiative for Transitioning Urban Systems towards Sustainable Futures, em uma publicação de 2007, propõe um quadro de referência para investigar a resiliência de sistemas urbanos. Neste trabalho, sugere-se uma perspectiva multinível, organizada em quatro espaços temáticos (Figura 3): i) fluxos metabólicos, ii) dinâmicas sociais, iii) estruturas de governança e iv) ambientes construídos. É no centro das quatro esferas de pesquisa que se posiciona a ideia de resiliência urbana.

Cada uma dessas esferas apresenta-se como um campo de exploração que se interliga com os demais no funcionamento dos sistemas urbanos. $\mathrm{O}$ balanço que resulta da consideração conjunta das quatro esferas produz o que poderemos designar por resiliência geral, a qual se adéqua melhor à natureza dos sistemas urbanos. Reconhece-se a preponderância dos fluxos metabólicos na sustentabilidade das funções urbanas, mas também no papel que desempenham enquanto fornecedores de bem-estar e qualidade de vida. As esferas de governança são centrais para que se internalize e difunda capacidade de aprendizagem coletiva, facilitadora de adaptações e que, por sua vez, sirva de catalisador para os diferentes desafios (Figura 3).

As dinâmicas sociais carregam nelas as ações das pessoas na sua condição de cidadãos, de participantes em comunidades, utilizadores de serviços, consumidores, fornecedores de produtos e serviços, mão de obra das empresas etc. A presença em cada um desses enquadramentos compõe fórmulas distintas de relacionamento com o ambiente construído, o qual, em diálogo constante, define os moldes físicos da forma 


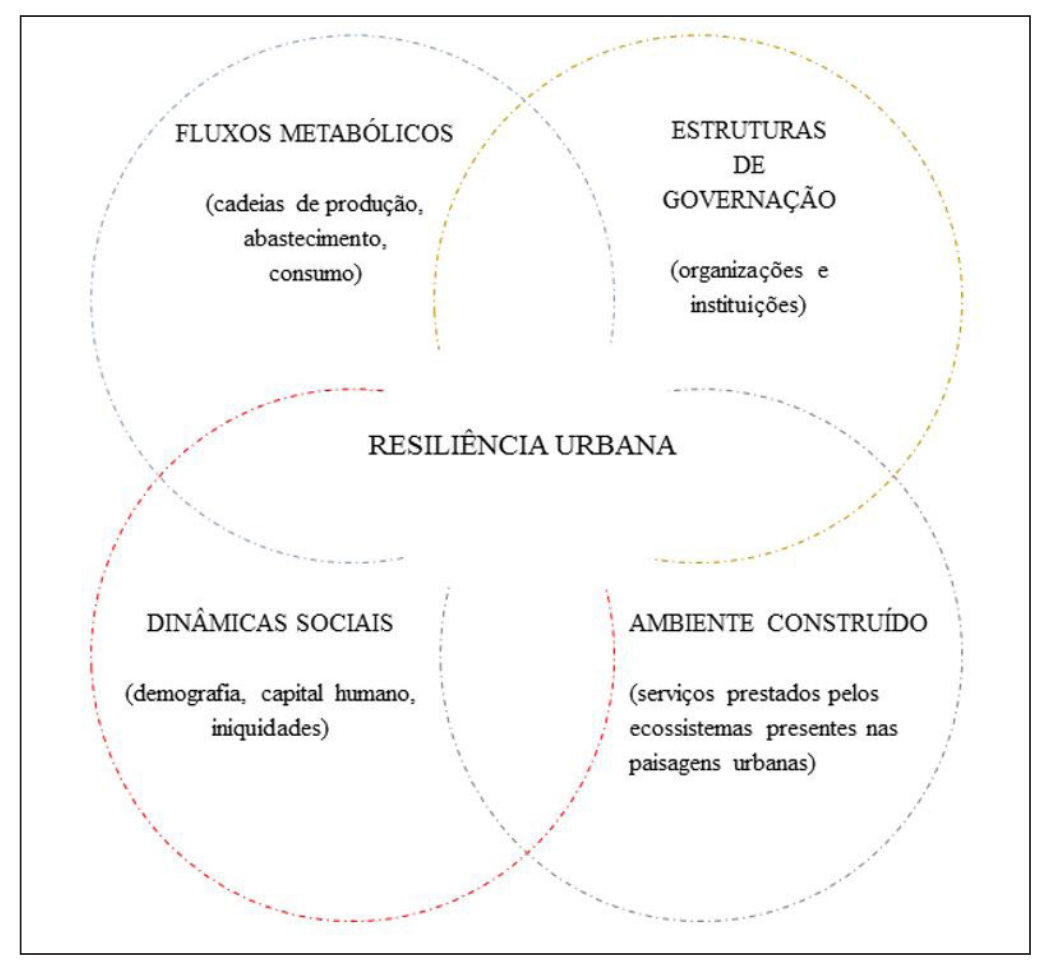

Figura 3 - Dimensões da resiliência urbana

Fonte: Adaptado de Resilience Alliance (2007, p. 10).

urbana e o respectivo espectro de relações e interconexões (Resilience Alliance, 2007). Tomadas individualmente, cada uma das componentes reproduzem quadros específicos de resiliência válidos, todavia parcelados.

Da noção de fluxo metabólico extrai-se uma parte das relações que os sistemas urbanos desenvolvem. Perspectiva-se a matriz de consumos associada às cadeias de produção geradas pelos ecossistemas, assim como as que estão presentes na base econômica. A relação entre maior ou menor dependência diante dos combustíveis fósseis (geografia das regiões produtoras, tipos de fontes, perfil de fornecedores), variações ou choques nesse plano e performance das atividades econômicas é um dos exemplos dessa interpenetração. Adicionalmente, a vulnerabilidade que resulta do fato de boa parte dos produtos manufaturados ter de viajar milhares de quilômetros entre as regiões de produção e de consumo, agudiza a estenose da economia assente no petróleo.

As sociedades industriais, em geral e as cidades em particular, são produtos do petróleo e podem implodir sem ele. Sem surpresa, a maior parte, senão mesmo todas as nossas cidades são insustentáveis [...] (Botequilha-Leitão, 2012, p. 14).
As retroações nas cadeias de produção e de consumo são indutoras de resiliência dos sistemas urbanos, na medida em que as cidades são sistemas abertos e a sua resiliência coincide com a das regiões fornecedoras de matérias-primas. Isso pode ser dito nos mesmos termos, mas pensando no sentido contrário. Avivam-se, assim, transfusões de responsabilidade entre produtores e consumidores, e entre consumidores e produtores. Entroncam-se aqui noções de autossuficiência parcial, de diversidade das cadeias de produção e consumo, de dependência perante fontes singulares de matérias-primas, de latitude das escolhas dos consumidores, de reciclagem dos recursos, de contágio entre escalas locais, regionais e globais, de estabilidade/instabilidade geopolítica e acessibilidade/inacessibilidade diante de centros monopolizadores de produção ou de consumo.

Na esfera das dinâmicas sociais (Figura 3), gravita a influência de aspectos como: ganhos e perdas demográficas, balanço entre criação/destruição de capital humano, fórmulas de distribuição ou captura dos recursos criados em uma comunidade. No tabuleiro demográfico, joga-se muito do potencial socioeconômico de um território: a capacidade natural para aumentar a população, para integrar 
redes de sistemas urbanos mais abrangentes, para atrair residentes de outras regiões, para estabelecer bases consistentes de acesso a serviços e amenidades públicas, e para sustentar desenvolvimento no longo prazo. Os quadros de vida dos indivíduos enquanto partes ativas das comunidades, células das cadeias de produção e consumo, utilizadores de serviços e geradores de efeitos de urbanidade, têm aqui um papel preponderante.

Nessa malha de interações, entendida como "ordem social" (Force \& Machlis 1997 apud Resilience Alliance 2007), relevam-se três canais: o primeiro compreende os aspectos de identidade mais pessoal (idade, gênero etc.); o segundo interioriza um conjunto de normas formais ou informais que baliza a convivência coletiva; o terceiro confere corpo às hierarquias presentes em uma sociedade e que estrutura, por exemplo, a acessibilidade ao poder político e/ou econômico. A resiliência como output agrega as ligações entre a ordem social, o funcionamento dos sistemas sociais e o potencial de capital humano disponível em um sistema urbano (Resilience Alliance, 2007). Nesse quadrante da análise, procura-se aderência, por exemplo, para a defesa da importância da coesão social na capacidade de resiliência das comunidades, para a relação entre tempos longos de deslocamento para o trabalho e menor tempo para as relações sociais e familiares, menor sentido de pertença a uma comunidade e, consequentemente, menor capital social.

Associam-se os fenômenos de segregação residencial à especialização localizada de modelos de construção que estratificam grupos, etnias e classes socais, como mecanismos de degradação da coesão e do capital social, expondo essas comunidades a maiores vulnerabilidades. São vários os elementos considerados nessa componente da resiliência urbana. Resumidamente, misturam-se aspectos da resiliência ou da vulnerabilidade dos propulsores demográficos com os difusores de equidade na distribuição da acessibilidade a recursos econômicos, culturais, educativos, de saúde, de participação na comunidade.

A inclusão da dimensão da governança na matriz, por meio da qual se perspectiva a resiliência urbana, conjuga o crescimento e o aumento da complexidade das cidades com o incremento de vulnerabilidade das instituições de gestão urbana, a emergência da dominância dos mercados e das forças da globalização, a tendência para a transferência e concentração de funções públicas para as esferas privadas, a remoção de barreiras ao comércio e ao investimento por algumas nações, agilizando os fluxos de bens, capital e informação entre fronteiras, transferindo centros de poder de governos nacionais para corporações e centros de poder globais e regionais (Resilience Alliance, 2007). Parte das normativas e das forças de decisão está, gradualmente, a ser exercida por instituições afastadas dos interesses das comunidades. Em consonância com esse quadro genérico, o desligamento das comunidades dos modos de participação cívica e política subtrai-lhes a capacidade de resiliência.

No avesso, as cidades com estruturas de governança robustas fomentam a distribuição do acesso a serviços pela população. Sem mecanismos ativos de redistribuição efetiva, a pobreza e a exclusão nos sistemas urbanos tendem a aprofundar-se (Pirez, 2002 apud Resilience Alliance, 2007). Sintetizando, os campos da "boa governança" ramificam-se pela: capacidade de prestar serviços de interesse geral; capacidade de gerir convenientemente os recursos financeiros disponíveis; competências para capitalizar a diversidade urbana, suprimir a fragmentação e a iniquidade; capacidade para atalhar ou aumentar a insegurança nos espaços urbanos; capacidade para gerar pontes e mecanismos de articulação para lidar com a crescente complexidade de autoridades, centros de poder e jurisdições (Resilience Alliance, 2007).

Equacionam-se conjuntamente a arquitetura da governança que produziu determinado grau de resiliência, quais as extensões emergentes e que efeitos terão. Coloca-se em questão a segmentação das componentes da governança urbana que mais influem na capacidade de resiliência dos sistemas urbanos e em que medida a qualidade das lideranças, da confiança, do capital social, da cristalização das instituições de poder, da estratificação das classes sociais interfere com a capacidade de resiliência. Consideram-se ainda as sobreposições de escalas territoriais e de poder público e privado, a reduzida interpenetração de esferas de cooperação, quando se observam sobreposições de escalas implicando na vulnerabilidade/resiliência urbanas. Por fim, procura-se saber em que circunstâncias os choques, as tensões e as crises incrementam vulnerabilidades para a malha da governação dos sistemas urbanos ou, pelo contrário, capitalizam a resiliência preexistente.

Em síntese, quando perspectivada no contexto dos sistemas urbanos, a resiliência pode assumir diferentes perfis. Considerando a magnitude e o ritmo 
das mudanças, as diferentes estruturas que compõem o sistema urbano atuam mais no sentido de procurar a estabilidade, ativando exclusivamente capacidade de absorção ou de resposta reativa, tentando fixar o modelo de desenvolvimento vigente. Pode associar a esse perfil a intrusão de fatores de flexibilidade que conferem capacidade adaptativa, relativizando o perfil de persistência com a possibilidade de introduzir adaptações incrementais. No melhor cenário, os dois perfis anteriores são atenuados pelo reforço da capacidade de introduzir mudanças e de injetar permanentemente transmutabilidade, reforçando o potencial para sustentar uma trajetória de desenvolvimento sustentável.

\section{Comunidades resilientes}

As comunidades, compostas por indivíduos, organizadas em famílias, em grupos, em estratos ou classes, são estruturas dinâmicas que criam oportunidades e que se deparam com desafios, alguns esperados, outros inesperados. A cada momento, as comunidades posicionam-se em torno de uma combinação de necessidades e dificuldades, recursos e oportunidades, ditadas por circunstâncias em constante atualização, integrando processos históricos com eventos em curso, definindo-se, assim, o seu futuro (Mancini \& Roberto, 2009). Para os autores, uma comunidade resiliente manifesta aptidão “[...] para enfrentar e adaptar-se a desafios e adversidades de maneira a promover, com sucesso, realizações que desloquem o seu modelo de desenvolvimento para um rumo desejável [...]" (Mancini \& Roberto, 2009, p. 248). Trata-se de um processo dinâmico que compreende adaptações positivas, desencadeadas também em situações de adversidade.

Enquanto atributo associável a uma comunidade, a resiliência conjuga vários conceitos. Assim, uma comunidade resiliente assimila essa característica como parte integrante da dinâmica social, conferindo-lhe condições de continuidade. Uma comunidade resiliente comporta mecanismos de adaptação diante de adversidades, pressupõe uma trajetória que viabiliza saldos positivos no capital social e ampliação das suas funcionalidades após um episódio de crise. 0 conjunto de marcadores que sinalizam tais propriedades deve viabilizar comparabilidade entre comunidades, posicionando umas por referência às outras (CARRI, 2013).
O documento referido anteriormente fornece um conjunto alargado de definições, propostas por vários autores ${ }^{3}$, que exploram a confluência entre resiliência e comunidade. De modo resumido, a resiliência das comunidades é entendida como capacidade para suportar choques nas suas infraestruturas sociais, capacidade de persistir mantendo e/ou ampliando a sua estrutura funcional, competências para gerar recursos que criem condições de funcionamento mais favoráveis. Compreende ainda um conjunto de ações que fomenta capacidades pessoais, coletivas e das instituições para responder e influenciar o curso das mudanças socioeconômicas.

A resiliência das comunidades pode ser de dois tipos: por um lado, pode ser vista como capacidade de o sistema socioeconômico facilitar os esforços para antecipar processos de mudança, reduzir vulnerabilidades e facilitar adaptação; por outro, corresponde à capacidade de o sistema socioeconômico suster e robustecer modelos de funcionamento profícuos.

Congregando as visões apontadas em pontos anteriores, uma comunidade resiliente: manifesta uma certa capacidade de absorver mudanças, mantendo o fulcro das suas funções, identidade, estruturas; apresenta soluções de auto-organização e de reorganização após um período de crise; demonstra capacidade de aprendizagem e de adaptação. Para além disso, demonstra capital coletivo para interpretar o ambiente socioeconômico e para desencadear mecanismos de adaptação. Mobiliza soluções para abandonar estádios indesejados, realocando os recursos disponíveis de modo eficiente e viabilizando trajetórias de desenvolvimento continuado. Assim, a resiliência de uma comunidade abre-se em três tipos de capacidade: de absorver o "novo" (novas populações, novas ideias, novos valores etc.); de mudar por meio de mecanismos que agilizam mutações institucionais; de acomodar o inesperado, desenvolvendo uma estrutura de planejamento apta a acolher imprevistos e colocar em causa a matriz de

\footnotetext{
${ }^{3} 0$ relatório de CARRI (2013) coleciona definições de várias fontes: Comfort (1999), Mileti (1999), Bruneau (2003), Godschalk (2003), Timmerman (1981), Wildavsky (1991), Brown (1996), Sonn (1998), Paton (2001), Center for Community Enterprise (2000), Chenoweth (2001), Ganor (2003), Kofinas (2003), Quinlan (2003), Ahmed (2004), Kimhi (2004), Coles (2004), Allenby (2005), Gunderson (2005), Pfefferbaum (2005), Subcommittee on Disaster Reduction (2005), UN/ISDR (2005), Norris (2008), Liu (2007), Perrings (2006).
} 
funcionamento corrente por referência a um quadro de incertezas (Within \& Boundaries, 2009).

Discutir a resiliência na escala das comunidades implica que se considere: o ponto de partida, ou seja, que se analise o status quo quanto aos resultados apresentados pré-crise (tempo 1); o quadro pós-crise, que compreende as consequências e os desafios que essa adversidade apresenta à comunidade (tempo 2); os resultados e o sentido das ações empreendidas pós-crise (tempo 3). Essas fases implicam a fluidez que só abordagens sistêmicas conseguem capturar. Assim, pode-se dizer que uma

[...] comunidade resiliente está habilitada a manter, reganhar, ou a estabelecer um programa de funcionamento satisfatório ou desejável ao longo do tempo, de forma a poder relativizar os contextos de adversidade. A sustentabilidade do seu modelo de desenvolvimento é tanto maior, quanto mais habilitada estiver para usar a adversidade no sentido de incrementar a resiliência (Mancini \& Roberto, 2009, p. 249).

Na ideia de organização social, cabe o conjunto de antecedentes que vincula uma comunidade a determinado contexto e as ações e os processos coletivos que conferem capital social. Esses elementos dialogam com um quadro de competências próprio da resiliência (Figura 4).

Segundo a designação de organização social de Mancini \& Roberto (2009), as condições interagem-se e características de uma comunidade com as redes e infraestruturas que facilitam a cooperação. Essas redes tanto podem ser de caráter formal como informal, mas todas potenciam mobilização. As relações familiares, o círculo de amizades, os espaços de sociabilidade associados aos lugares de trabalho são alguns exemplos de ligações que desenham redes. Redes que, de acordo com a respectiva densidade de ligações, criam padrões de resiliência nas respectivas comunidades. Muito do capital social é forjado nesse emaranhado de fluxos por onde circula informação, reciprocidade e confiança, e que, por sua vez, faz emergir um conjunto de recursos sociais. As capacidades de uma comunidade podem ser vistas no sentido de responsabilidade, no envolvimento coletivo, na partilha de crenças ou no perfilhar de objetivos que projetem qualidade de vida no longo prazo. Podem ainda traduzir competências para identificar oportunidades de restabelecimento do capital social perdido ou fazê-lo progredir, por oposição à concepção de ações reativas diante dos riscos. Esse padrão de comportamento manifesta-se no quotidiano e reafirma-se em situações ameaçadoras

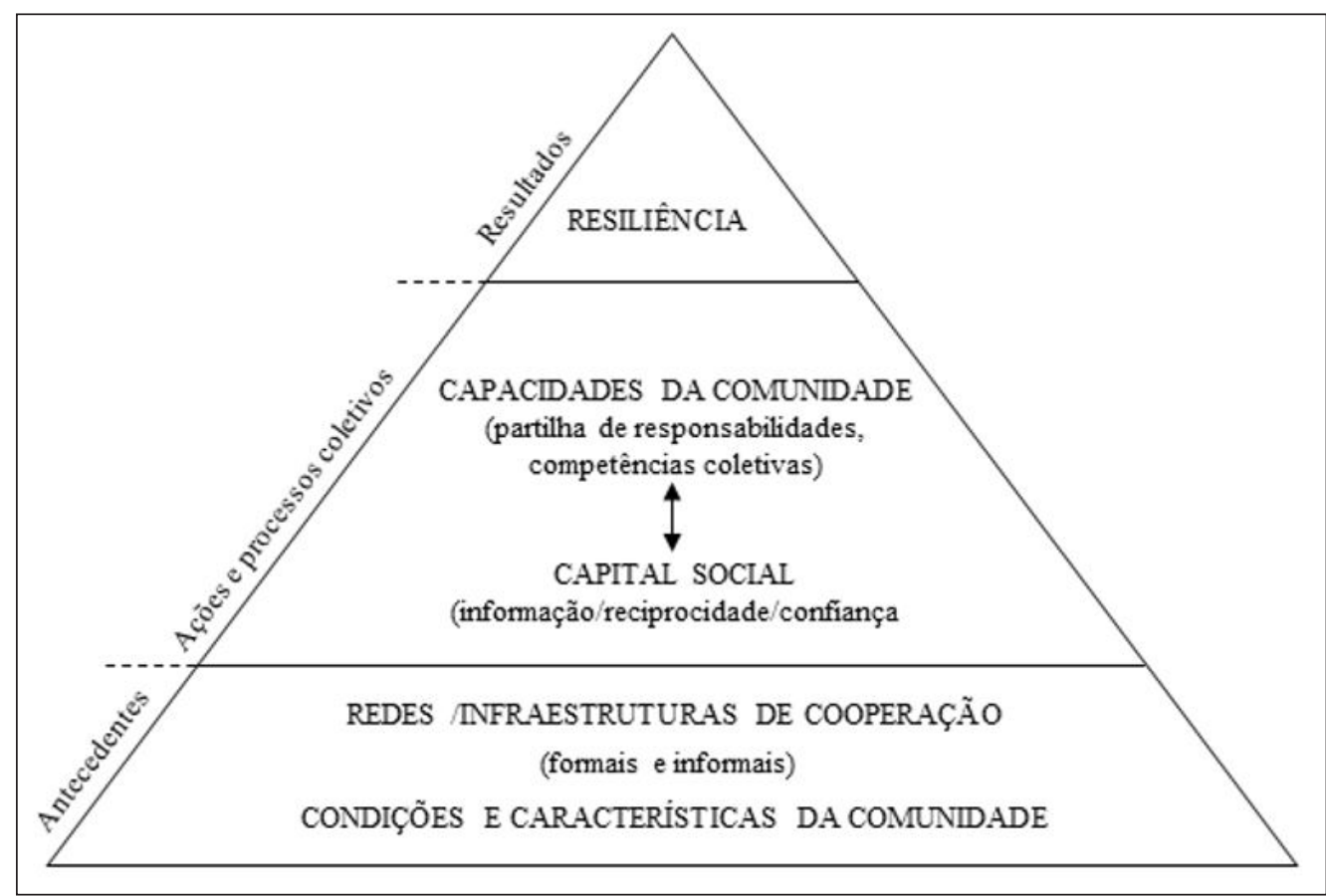

Figura 4 - Organização social e condiç̃oes de mudança Fonte: Adaptado de Mancini \& Roberto (2009). 
ou de crise. 0 nível de conhecimentos que caracteriza uma comunidade, os seus círculos de interações, o seu capital de confiança, o nível de coesão e a latitude dos espaços de cooperação configuram uma dada organização social, que, por sua vez, apresenta determinado alcance enquanto alavancas de resiliência (Mancini \& Roberto, 2009).

\section{Conclusão}

Uma ruptura na circulação rodoviária pode, de um minuto para o outro, bloquear uma cidade. Uma descontinuidade nas transações no mercado financeiro pode fazer colapsar a estrutura econômica no decurso de um dia ou de uma semana. Um corte na linha de valorização do mercado imobiliário pode inverter a trajetória de desenvolvimento de uma cidade, com extensões à escala regional e até mesmo global, no decorrer de alguns meses ou em poucos anos. Uma alteração repentina no preço dos combustíveis ou dos alimentos (por exemplo, dos cereais) é passível de provocar efeitos semelhantes. Alargando a escala do tempo, ao se pensar na consolidação de um processo de gentrificação, podemos ter de considerar intervalos de décadas nos quais uma área da cidade reconfigura a sua matriz. Note-se ainda que mudanças abruptas em um sistema podem decorrer de alterações graduais, porventura insignificantes, por referência a outro sistema organizado em torno de uma escala de grandeza diferente. Importa dizer que as rupturas a que as cidades estão sujeitas dependem tanto da escala temporal como da geográfica.

Para tal, é fulcral que se identifiquem questões nucleares, como saber identificar e fazer germinar focos de transformação, ou qual a relevância da interpenetração de escalas geográficas na criação de resiliência evolutiva, que, por sua vez, catapulte sustentabilidade. Está em causa o entendimento da resiliência como uma capacidade que as regiões, as cidades, as organizações ou as comunidades podem internalizar.

Uma região (tal como uma cidade ou uma comunidade) resiliente desenvolve um modelo de progresso assente em mudanças graduais e continuadas. Esse território amplia oportunidades para todos os grupos etários e sociais, estabelece e consolida uma rede de conectividades e internaliza, na sua matriz socioeconômica, condições de aprendizagem que permitem evitar ou inovar a partir de quadros de tensão. Para além disso, posiciona-se de modo a integrar sistemas territoriais que potenciem interações em múltiplas escalas. No centro das atuações, está a necessidade de facilitar uma cultura de resiliência nas esferas do estado, das organizações e dos indivíduos.

Agregando as abordagens apresentadas e discutidas nos pontos anteriores, é possível afirmar que regiões, cidades e comunidades resilientes minimizam os efeitos das crises e demonstram competências para recuperar os pontos nevrálgicos da sua vitalidade socioeconômica quando não conseguem evitá-las. Em síntese: uma comunidade resiliente, não obstante os episódios de crises, conserva, reganha ou estabelece resultados favoráveis ao longo do tempo, desencadeando ininterruptamente mudanças profícuas nos quadros de vidas quotidianos.

\section{Referências}

Adger, N. (2000). Social and ecological resilience: are they related? Progress in Human Geography, 24(3), 347-364. http://doi.org/10.1191/030913200701540465.

Adger, N., Hughes, T., Folke, C., Carpenter, S., \& Rockström, J. (2005). Social-ecological resilience to coastal disasters. Science, 309(5737), 1-6. http://doi.org/10.1126/ science.1112122.

Alberti, M., Marzluff, J. M., Shulenberger, E., Bradley, G., Ryan, C., \& Zumbrunnen, C. (2003). Integrating humans into ecology: opportunities and challenges for studying urban ecosystems. Bioscience, 53(12), 1169-1179. http://dx.doi. org/10.1641/0006-3568(2003)053[1169:IHIEOA]2.0.CO;2.

Ballard-Rosa, G. (2010). Book review: "what is a city? Rethinking the urban after hurricane katrina". Critical Planning, 17, 174-181.

Batty, M., Barros, J., \& Alves, S., Jr. (2004). Cities: continuity, transformation, and emergence (CASA Working Paper Series, No 72). London: Centre for Advanced Spatial Analysis (CASA), University College.

Berke, P. R., \& Campanella, T. J. (2006). Planning for postdisaster resiliency. The Annals of the American Academy of Political and Social Science, 604(1), 192-207. http:// dx.doi.org/10.1177/0002716205285533.

Botequilha-Leitão, A. (2012). Eco-polycentric urban systems: an ecological region perspective for network 
cities. Challenges, 3(1), 1-42. http://dx.doi.org/10.3390/ challe3010001.

Brock, W. A., Mäler, K., \& Perrings, C. A. (2002). Resilience and sustainability: the economic analysis of non-linear dynamic systems. In L. Gunderson, D. Ludwig, \& C. S. Holling (Eds.), Panarchy: understanding transformations in human and natural systems (p. 261-292). Washington: Island Press.

Carpenter, S., Walker, B., Anderies, M., \& Abel, N. (2001). From metaphor to measurement: resilience of what to what? Ecosystems, 4(8), 765-781. http://doi.org/10.1007/ s10021-001-0045-9.

Chapple, K., \& Lester, T. W. (2010). The resilient regional labour market? The US case. Cambridge Journal of Regions, Economy and Society, 3(1), 85-104. http://dx.doi. org/10.1093/cjres/rsp031.

Chelleri, L. (2012). From the resilient city to urban resilience: a review essay on understanding and integrating the resilience perspective for urban systems. Documents d'Analisi Geografica, 58(2), 287-306. http://dx.doi.org/10.5565/ rev/dag.175.

Community \& Regional Resilience Institute - CARRI. (2013). Definitions of community resilience: an analysis. Washington, DC: CARRI.

Cumming, G. S., Barnes, G., Perz, S., Schmink, M., Sieving, K. E., Southworth, J., Binford, M., Holt, R. D., Stickler, C., \& Van Holt, T., (2005). An exploratory framework for the empirical measurement of resilience. Ecosystems, 8, 975-987.

Dabson, B., Heflin, C. M., \& Miller, K. K. (2012). Regional resilience: research and policy brief. Missouri: University of Missouri.

Dawley, S., Pike, A., \& Tomaney, J. (2010). Towards the resilient region? Local Economy, 25(8), 650-667. http:// dx.doi.org/10.1080/02690942.2010.533424.

Dempster, C. (2007). Resilience of Social-Ecological Systems (SESs): a case study of water management in the Iraqi Marshlands. Kingston: Queen's University.

ESPON. (2012). Economic crisis: resilience of regions (Inception report). Luxembourg: ESPON.

Ficenec, S. (2010). Building regional economic resilience: what can we learn from other fields? Washington, DC: George Washington Institute of Public Policy, George Washington University.
Folke, C. (2006). Resilience: the emergence of a perspective for social-ecological systems analyses. Global Environmental Change, 16(3), 253-267. http://doi.org/10.1016/j. gloenvcha.2006.04.002.

Folke, C., Carpenter, S., Elmqvist, T., Gunderson, L., Holling, C. S., \& Walker, B. (2002). Resilience and sustainable development: building adaptive capacity in a world of transformations. Ambio, 31(5), 437-440. Recuperado em 09 de março 2016, de http://www.ncbi.nlm.nih.gov/ pubmed/12374053.

Folke, C. S., Carpenter, S. R., Walker, B., Scheffer, M., Elmqvist, T., Gunderson, L., \& Holling, C. S. (2004). Regime shifts, resilience, and biodiversity in ecosystem management. Annual Review of Ecology and Evolution, 35, 557-581. http://doi.org/10.1146/annurev.ecolsys.35.021103.105711.

Godschalk, D. R. (2003). Urban hazard mitigation: creating resilient cities. Natural Hazards Review, 4(3), 136-143. http://dx.doi.org/10.1061/(ASCE)1527-6988(2003)4:3(136).

Holling, C. S. (1973). Resilience and stability of ecological systems. Annual Review of Ecology and Systematics, 4, 1-23.

Holling, C. S. (2001). Understanding the complexity of economic, ecological, and social systems. Ecosystems (New York, N.Y.), 4(5), 390-405. http://dx.doi.org/10.1007/ s10021-001-0101-5.

Holling, C. S., \& Meffet, G. K. (1996). Command and control and the pathology of natural resource management. Conservation Biology, 10(2), 328-337.

Mancini, J., \& Roberto, K. (2009). Pathways of human development: explorations of change. In J. Mancini, \& K. Roberto (Eds.), Community resilience: a social organization theory of action and change (p. 245-265). New York: Lexington Books.

Martin, R. (2012). Regional economic resilience, hysteresis and recessionary shocks. Journal of Economic Geography, 12(1), 1-32. http://dx.doi.org/10.1093/jeg/lbr019.

Martin-Breen, P., \& Anderies, J. M. (2011). Resilience: $a$ literature review. New York: Cunty Graduate Center; Tempe: Arizona State University.

Naudé, W., Mcgillivray, M., \& Rossouw, S. (2009). Measuring the Vulnerability of Subnational Regions. Oxford Development Studies, 37(3), 249-276. http://dx.doi. org/10.1080/13600810903085800.

Organização das Nações Unidas - ONU. (2012). Povos resilientes, planeta resiliente: um futuro digno de escolha 
(Painel de Alto Nível do Secretário-Geral das Nações Unidas sobre Sustentabilidade Global). New York: ONU.

Ott, K., \& Döring, R. (2004). Theorie und Praxis starker Nachhaltigkeit. Marburg: Metropolis Verlag.

Parr, J. B. (2014). The regional economy, spatial structure and regional urban systems. Regional Studies, 48(12), 1926-1938.

Pendall, R., Foster, K., \& Cowell, M. (2010). Resilience and regions: building understanding of the metaphor. Cambridge Journal of Regions, Economy and Society, 3(1), 71-84. http://dx.doi.org/10.1093/cjres/rsp028.

Perrings, C. (2006). Resilience and sustainable development. Environment and Development Economics, 11(4), 417-427. http://doi.org/10.1017/S1355770X06003020.

Pickett, S., Cadenasso, M., \& Grove, J. (2004). Resilient cities: meaning, models, and metaphor for integrating the ecological, socio-economic, and planning realms. Landscape and Urban Planning, 69, 369-384. http://doi. org/10.1016/j.landurbplan.2003.10.035.

Raco, M., \& Street, E. (2012). Resilience planning, economic change and in London and Hong Kong. Urban Studies (Edinburgh, Scotland), 49(5), 1065-1087. http://dx.doi. org/10.1177/0042098011415716.

Resilience Alliance. (2007). A research prospectus for urban resilience. Recuperado em 09 de março 2016, de http://www.resalliance.org/files/1172764197_ urbanresilienceresearchprospectusv7feb07.pdf

Robert, C. (2001). The polycentric urban region: towards a research agenda. Urban Studies (Edinburgh, Scotland), 38(4), 623-633. http://dx.doi.org/10.1080/00420980120035259.

Simmie, J., \& Martin, R. (2010). The economic resilience of regions: towards an evolutionary approach. Cambridge Journal of Regions, Economy and Society, 3(1), 27-43. http:// dx.doi.org/10.1093/cjres/rsp029.

Vale, L. J., \& Campanella, T. H. (Eds.). (2005). The resilient city: how modern cities recover from disaster. New York: Oxford University Press.

Walker, B., \& Salt, D. (2006). Resilience thinking, sustaining ecosystems and people in a changing world. Washington, D.C.: Island Press.

Walker, B., Carpenter, S., Anderies, J., Abel, N., Cumming, G., Janssen M., Lebel, L., Norberg, J., Peterson G. D., \& Pritchard, R. (2002). Resilience management in social-ecological systems: a working hypothesis for a participatory approach. Ecology and Society, 6(1), 14.

Within, R. C., \& Boundaries, A. (2009). Community resilience: a cross-cultural study revitalizing community within and across boundaries. Washington, DC: Woodrow Wilson International Center for Scholars.

Recebido: Mar. 09, 2016

Aprovado: Ago. 01, 2016 